\title{
Fractional-order scheme for bovine babesiosis disease and tick populations
}

\author{
Zain UI Abadin Zafar ${ }^{1,2^{*}}$, Kashif Rehan ${ }^{3}$ and M Mushtaq ${ }^{1}$
}

"Correspondence:

zainzafar2016@hotmail.com

${ }^{1}$ Department of Mathematics,

University of Engineering and

Technology, Lahore, Pakistan

${ }^{2}$ Faculty of Information Technology,

University of Central Punjab, Lahore,

Pakistan

Full list of author information is

available at the end of the article

\begin{abstract}
This article shows epidemic model, earlier suggested in ordinary lifferential equation philosophy, can be extended to fractional order on a reliable lage ' comportment. A set of domains for the model wherein al'va. ves ar IImited is established. Furthermore, the stability and existence of steadint noints are studied. We present the evidence that the endemic equilibr um poin is locally asymptotically stable when reproduction numbor $R_{0}>1$. outcome is attained via the linearization statement for fractional diffe ent s, equations (FDEs). The worldwide asymptotic stability of a disease-free point, $w_{1}$, is also verified by comparison theory for fractional differential equations. The $n_{c}$ aric replications for diverse consequences are carried out, and data an an are in good agreement with theoretical outcomes, displaying a vital psicepuion about the use of the set of fractional coupled differential enıations ti model babesiosis disease and tick populations.
\end{abstract}

Keywords: bovine b-hesio sta ility; predictor-corrector technique; reproduction number

\section{Intrc duction}

Bovine besiosi, (BB) is communicated by the bite of ticks and is the most important disense to atm Dovine populations in humid areas. In hot and warm areas there is great financ. $s$ due to bovine death by $\mathrm{BB}$, with decrease of bovine products and by-products. Bes des, the environment conditions in those regions favor the survival and reproduction $c$ ticks, so bovines have an enduring interaction with these vectors [1]. What is more, the vertical spread in bovines and ticks is likely to happen when the ovaries of the female ticks are plague-ridden by parasites [1]. The behavior dynamics of syndromes has been considered for a stretched period and is an important issue in the real world. The most important model that can be used to interpret the disease characteristic of epidemics is the susceptible-infected-recovered (SIR) model that was developed by Kermack and McKendrick [2]. Various types of diseases are studied by this type of ordinary differential equation system. Aranda et al. [3] introduced the epidemiological model for bovine babesiosis and tick populations disease. In this work the qualitative dynamics behavior is determined by the reproduction number $R_{0}$. If the threshold parameter $R_{0}<1$ is proved by the LaSalle-Lyapunov theorem, then the solution converges to the disease free equilibrium (DFE) point. However, if $R_{0}>1$, the merging is to the EE point by numerical imitations. In

\section{Springer}


recent years, the theory of networks epidemiological model has been introduced in the literature. The purpose of this modification is to have a better understanding and prediction of epidemic patterns and intervention measures. For more details, see [4-6].

Leibniz, one of the originators of ordinary calculus, introduced the concept of fractional calculus in a memo transcribed in 1695. In latest eras, FDEs have become one of the most important topics in mathematics and have received much consideration and growing curiosity due to the options of unfolding nonlinear systems and due to their prospective applications in physics, control theory, and engineering [7-15]. The benefit of FDE syc tems is that they allow greater degrees of freedom and incorporate the memory effe' $t$ in the model. Due to this fact, they were introduced in epidemiological modeling ystems. In [16], a fractional order for the dynamics of A (H1N1) influenza disease wos st "ied by numerical simulations. Pooseh et al. [17] and Diethelm [18] introduced fract. al dengue models. In this article the parameters of equations obtained in the fo researc not reproduce well the evolution of the disease in the case of entire order is del. However, when we consider the fractional system with the same param - $\mathrm{e}_{2}$ obtaine $\mu$ in the field, the data are better adjusted, which shows an advantage of th il system. In [11] the parameter $\theta$ is associated with a memory effect. In [19], the a ors attribute to $\theta$ the memory information of the dengue diseases. In this articic, ponder on the fractional order system linked with the development of BB disease and tick populations. We introduce a broad view of the classical model prescrito Aranda et al. [3]. The generalization is attained by changing the ordinary derivat. with he fractional Caputo derivative. It is easy to see that when $\theta=1$ we return to the cla al model. For the construction of this model by Aranda et al. [3], the compa nents populations and the biological hypothesis are used. This argument is $1 \mathrm{establ}$ - d in the disease transmission theory. Aranda et al. use theorems well estaulish in the literature for ordinary differential systems. To prove our results, it is $r$ ecessary to use tools different from those used for the integer order. This is due to the $f$ that th e versions of La-Salle invariance theorem used by Aranda et al. are not found in the ature for fractional-order systems. Therefore, we emphasize that the work prest. Haboration in this direction when using the comparison theory for fracti -orde systems to verify the worldwide stability of DFE point of the disease by int 'uc new type of results in the literature. On the other hand, we also have a est on $\downarrow$ native asymptotic stability of EE point, a result that was just enunciated by A. Ja et al. [3]. We obtain a generalization of all results in [3]. Our simulation shows that the fra conal model has great potential to describe the real problem without the need for ad ustment of parameters obtained in the field research. This is due to a greater flexibility of adjustment obtained with the introduction of the new parameter.

Fractional calculus represents a generalization of the ordinary differentiation and integration to non-integer and complex order [20]. The generalization of differential calculus to non-integer orders of derivatives can be traced back to Leibnitz [21]. The main reason for using integer order models was the absence of solution methods for fractional differential equations. It is an emerging field in the area of applied mathematics and mathematical physics such as chemistry, biology, economics, image, and signal processing, and it has many applications in many areas of science and engineering [22], for example, viscoelasticity, control theory, heat conduction, electricity, chaos and fractals, etc. [20]. Various applications, like in the reaction kinetics of proteins, the anomalous electron transport in 
amorphous materials, the dielectrical or mechanical relation of polymers, the modeling of glass forming liquids and others, are successfully performed in numerous papers [21].

The physical and geometrical meaning of the non-integer integral containing the real and complex conjugate power-law exponent has been proposed. Finding examples of real systems described by the fractional derivative is an open issue in the area of fractional calculus [20]. Since integer order differential equations cannot precisely describe the experimental and field measurement data, as an alternative approach, non-integer order differential equation models are now being widely applied [23, 24]. The advantage of fractional-order differential equation systems over ordinary differential equation system. is that they allow greater degrees of freedom and incorporate memory effect in the model. In other words, they provide an excellent tool for the description of memory an hereditary properties which were not taken into account in the classical intege: del do? [25]. The calculus of variations is widely applied for some disciplines ilke e ${ }_{{ }_{2}}$ 'eering, pure and applied mathematics. Moreover, the researchers have rec $\mathrm{n}_{\mathrm{L}}$. vroved nat the physical systems with dissipation can be clearly modeled more arcurately using fractional representations [22]. Recently, most of the dynamical s cten based on the integer order calculus have been modified into the fractional order do in aue to the extra degree of freedom and the flexibility which can be used to asely fit ne experimental data much better than in the case of the integer order modelling

Purohit and Kalla [26] discussed the generalized fractional partial differential equations involving the Caputo time-fractional derivat ve an the Liouville space-fractional derivatives. The solutions of these equations are ob. ned $\mathrm{c}$,ing Laplace and Fourier transforms. Also Purohit [27] discussed the gener lized fract al partial differential involving the Hilfer time-fractional derivative and the ce-fr ctional generalized Laplace operators occurring in quantum mechanir Chouha $t$ al. [28] presented the method for deriving the solution of the generalized for of fractional differential equation and Volterra-type differential equation. $\mathrm{N}$ sar et al. [29/1 discussed a generalized fractional kinetic equation involving generalized ssel function of the first kind. Also some of interesting nonlinear models and fractional $\mathrm{n}$. 1 - have been discussed in [30-33].

This article is wo red in four segments. Introduction is the first segment in which we elabor on sc ne history of fractional calculus. In Section 2, we elaborate notations relater o th conc pt of FDEs. In Section 3, we ponder on the fractional-order model lin'cd w the aynamics of bovine babesiosis and tick populations. Qualitative dynamics o. 'e mode, are resoluted by the elementary reproduction number. We provide a compreh iye investigation for the global asymptotical stability of DFE point and the native asymptotical stability of EE point. In Section 4, numerical imitations are offered to validate th main outcomes, and finally conclusion is drawn in Section 5.

\section{Preliminaries}

For several ages, there have been numerous definitions that fit the notion of fractional derivatives [10,34]. In this article the Riemann-Liouville fractional derivative, the Caputo fractional derivative, and Grunwald-Letnikov definitions are presented. Firstly, we introduce the definition of Riemann-Liouville fractional integral

$$
J^{\varphi} g(x)=\frac{1}{\Gamma(\varphi)} \int_{0}^{x}(x-s)^{\varphi-1} g(s) d s
$$

where $\varphi>0, f \in L^{1}\left(R^{+}\right)$, and $\Gamma(\cdot)$ is gamma function. 
The Riemann-Liouville derivative is given by

$$
D_{R}^{\varphi} g(x)=\frac{d^{m}}{d x^{m}}\left[J^{m-\varphi} g(x)\right]=\frac{1}{\Gamma(m-\varphi)} \frac{d^{m}}{d x^{m}} \int_{0}^{x}(x-s)^{m-\varphi-1} g(s) d s, \quad m-1 \leq \varphi<m .
$$

The Caputo fractional derivative (CFD) is agreed to be as follows:

$$
D_{C}^{\varphi} g(x)=J^{m-\varphi}\left[\frac{d^{m}}{d x^{m}} g(x)\right]=\frac{1}{\Gamma(m-\varphi)} \int_{0}^{x}(x-s)^{m-\varphi-1} g^{(m)}(s) d s,
$$

where $m$ is the first integer not less than $\varphi$.

The Grunwald-Letnikov derivative is given by

$$
{ }_{a} D_{x_{k}}^{\varphi} g(x)=\lim _{h \rightarrow 0} \frac{1}{h^{\varphi}} \sum_{j=0}^{\left[\frac{x-a}{h}\right]}(-1)^{j}\left(\frac{\varphi}{j}\right) g(x-j h),
$$

where $[\cdot]$ means the integer part.

The Laplace transform of the CFD is specified by

$$
\mathcal{L}\left[D_{C}^{\varphi} g(x)\right]=s^{\varphi} G(s)-\sum_{j=0}^{n-1} g^{(j)}(0) s^{\varphi-j-1} .
$$

The Mittag-Leffler function is defined by the fo yng infinite power series:

$$
E_{\alpha, \beta}(z)=\sum_{k=0}^{\infty} \frac{z^{k}}{(\alpha k+\beta)}
$$

The Laplace transforn of the functions is

$$
\mathcal{L}\left[t ^ { \beta - 1 } E _ { \alpha , \beta } \left(=w_{v}, \frac{s^{\alpha-\beta}}{s^{\alpha} \mp a} .\right.\right.
$$

Let $\alpha, 0,-\leq \mathbb{C}$, and the Mittag-Leffler functions satisfy the equality given by Theorem 4.2 , 10]

$$
E_{y, \beta}(z)=z E_{\alpha, \alpha+\beta}(z)+\frac{1}{\Gamma(\beta)} .
$$

Demarcation 1 A function $F$ is Holder continuous if there are non-negative amounts $G$, $v$ such that

$$
\|F(u)-F(v)\| \leq G\|u-v\|^{v}
$$

for all $u, v$ in the purview of $F$ and $v$ is the Holder exponent. We represent the space of Holder-continuous functions by $G^{0, v}$.

We improve a generalized inequality, in which the core appraisal system is a vector fractional order system. 
A non-negative (resp., positive) vector $v$ means that each constituent of $v$ is nonnegative (resp., positive). We represent a non-negative (resp., positive) vector by $0 \leq \leq v$ (resp., $0 \ll v)$.

Consider the fractional order system

$$
D_{C}^{\varphi} w(t)=g(t, w)
$$

with the initial condition $w(0)=w_{0}$, where $D_{C}^{\varphi} w(t)=\left(D_{C}^{\varphi} w_{1}(t), D_{C}^{\varphi} w_{2}(t), D_{C}^{\varphi} w_{3}(t), .\right.$. $\left.D_{C}^{\varphi} w_{m}(t)\right)^{H}, 0<\varphi<1, w(t) \in \mathcal{F} \subset R^{m}, t \in[0, H)(H \leq \infty), \mathcal{F}$ is an open set, $0 \in \mathcal{F}$, anc $g:[0, H) \times \mathcal{F} \rightarrow R^{m}$ is continuous in $t$ and mollifies the Lipschitz condition

$$
\left\|g\left(t, w^{\prime}\right)-g\left(t, w^{\prime \prime}\right)\right\| \leq W\left\|w^{\prime}-w^{\prime \prime}\right\|, \quad t \in[0, H)
$$

for all $w^{\prime}, w^{\prime \prime} \in \Omega \subset \mathcal{F}$, where $W>0$ is a Lipschitz constant.

Theorem 1 (see [15]) Let $u(t), t \in[0, H)$, be the solution of sys $n$ (1). If there exists a vectorfunction $w=\left(w_{1}, w_{2}, \ldots, w_{m}\right)^{H}:[0, H) \rightarrow \mathcal{F}$ such that $w_{i} \in G \quad n<w<1, i=1,2, \ldots, m$, and

$$
D_{C}^{\varphi} w \leq \leq g(t, w), \quad t \in[0, H]
$$

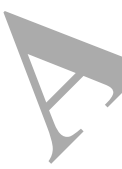

If $w(0) \leq \leq u_{0}, u_{0} \in \mathcal{F}$, then $w \leq \leq u, t \in\left[0, H_{\perp}\right.$

Let $g: \mathcal{F} \rightarrow R^{m}, \mathcal{F} \in R^{m}$, we considel toll ving system of fractional order:

$$
D_{C}^{\varphi} x(t)=g(x), \quad x(0)=x_{\partial}
$$

Demarcation 2 We $\mathrm{s}$ that $E \mathrm{i}$, an equilibrium point of (13) if and only if $g(E)=0$.

Remark 1 Whel (n.1), the fractional system $D_{C}^{\varphi} x(t)=g(x)$ has identical equilibrium points as the arran oment $x^{\prime}(t)=g(x)$.

Def nit 3 tre equilibrium point $E$ of autonomous system (13) is said to be stable if for $>0, \varepsilon>$ exists such that if $\left\|x_{0}-E\right\|<\varepsilon$, then $\|x-E\|<\epsilon, t \geq 0$; the equilibrium point $E$ or tonomous system (13) is said to be asymptotically unwavering if $\lim _{t \rightarrow \infty} x(t)=E$.

T) eorem 2 ([12]) The equilibrium points of system (13) are locally asymptotically stable If all eigenvalues $\lambda_{\mathrm{i}}$ of the Jacobian matrix $J$, calculated in the equilibrium points, satisfy $\left|\arg \left(\lambda_{\mathrm{i}}\right)\right|>\varphi \frac{\pi}{2}$.

\section{Mathematical model}

In this segment, we introduce the fractional model for the BB in bovine and tick populations. We use the assumptions in Aranda et al. [3] and introduce the following hypotheses.

(i) The total of bovine population $T_{B}(t)$ is distributed into three-fold sub-populations:

(a) the susceptible $X_{B}(t)$ that can turn into infected;

(b) the infected $Y_{B}(t)$, that is, bovines infected by Babesia parasite; 
(c) the recovered or controlled $Z_{B}(t)$ that have been cured.

(ii) The birth rate factor of bovine is represented by $\mu_{B}$. The birth rate $\mu$ is presumed to be equal to the normal demise.

(iii) $T_{T}(t)$ is the entire population of ticks that is distributed into two-fold sub-populations:

(a) $X_{T}(t)$ is tick population which may become infected by the disease;

(b) ticks infected by Babesia parasite are represented by $Y_{T}(t)$.

(iv) The birth rate factor of ticks is represented by $\mu_{T}$, and it is presumed to be equal to the normal demise rate.

(v) A susceptible bovine can move to the infected sub-population $Y_{B}(t)$ as of $\mathrm{n}$ effective transmission due to a sting of an infected tick at rate $\beta_{B}$.

(vi) A susceptible tick may be infected if there exists an active diffusion wh it bites a diseased bovine at rate $\beta_{T}$.

(vii) We presumed a hundred percent vertical diffusion in the bovine $\mathrm{p}$ lation $\mu_{B}$. In the tick population it befalls with possibility $1-p$, where $p$. the possibility that a susceptible tick was born from an infected one.

(viii) A fraction $\lambda_{B}$ of the diseased bovine is controlle' i.e., free in Babesia parasite.

(ix) A fraction $\alpha$ of the controlled bovine can yield to the eptible state, $\alpha \in(0,1)$.

(x) Identical involvement is presumed, i.e., all susceptile bovines have equal possibility to the diseased, and all suc cepu ticks have equal possibility to the diseased.

In the above conventions, the trans lission dyi hics of babesiosis disease to bovine and tick populations can be modeled $1 \mathrm{v}$ t. . Illow ng system [3]:

$$
\left\{\begin{array}{l}
X_{B}^{\prime}=\mu_{B}\left(X_{B}+Z_{B}\right)+Z_{B}-\mu_{L}-\beta_{B} X_{B} \frac{Y_{T}}{T_{T}}, \\
Y_{B}^{\prime}=\mu_{B} Y_{B}+\beta_{B} \quad{ }_{B} \frac{Y_{T}}{T_{T}}-\left(\mu_{B}+\lambda_{B}\right) Y_{B}, \\
Z_{B}^{\prime}=\lambda_{B} Y_{B}-\left(\mu_{B}\right) \\
X_{T}^{\prime}=\mu_{T}\left(\lambda_{T}-\mu_{T} X_{T}-\beta_{T} X_{T} \frac{Y_{B}}{T_{B}}\right. \\
Y_{T}^{\prime} \mu_{T} Y_{T} \pm \beta_{T} X_{T} \frac{Y_{B}}{T_{B}}+(1-p) Y_{B} .
\end{array}\right.
$$

\section{Fract, 9 order model}

In - rent years, a substantial attention to the fractional calculus has been shown, which allows, as to consider integration and differentiation of any order. To a large extent, this is di to the uses of fractional calculus to problems in different areas of research. The benefit of FDE systems is that they allow greater degrees of freedom and incorporate memory effect in the model. Now we describe a new system of FDEs to model the babesiosis disease in bovine and tick populations, and in this system $\phi \in(0,1)$.

$$
\left\{\begin{array}{l}
D^{\phi_{1}} X_{B}=\mu_{B}\left(X_{B}+Z_{B}\right)+\alpha Z_{B}-\mu_{B} X_{B}-\beta_{B} X_{B} \frac{Y_{T}}{T_{T}} \\
D^{\phi_{2}} Y_{B}=\mu_{B} Y_{B}+\beta_{B} X_{B} \frac{Y_{T}}{T_{T}}-\left(\mu_{B}+\lambda_{B}\right) Y_{B} \\
D^{\phi_{3}} Z_{B}=\lambda_{B} Y_{B}-\left(\mu_{B}+\alpha\right) Z_{B} \\
D^{\phi_{4}} X_{T}=\mu_{T}\left(X_{T}+p Y_{T}\right)-\mu_{T} X_{T}-\beta_{T} X_{T} \frac{Y_{B}}{T_{B}} \\
D^{\phi_{5}} Y_{T}=-\mu_{T} Y_{T}+\beta_{T} X_{T} \frac{Y_{B}}{T_{B}}+(1-p) Y_{B} .
\end{array}\right.
$$


Simplifying system (15), using the bovine and tick populations' constants $T_{B}$ and $T_{T}$, respectively, and introducing the proportions

$$
U=\frac{X_{B}}{T_{B}}, \quad V=\frac{Y_{B}}{T_{B}}, \quad W=\frac{Z_{B}}{T_{B}}, \quad X=\frac{X_{T}}{T_{T}}, \quad Z=\frac{X_{T}}{T_{T}},
$$

we attain the following fractional system that defines the dynamics of bovine quantity in each class:

$$
\left\{\begin{array}{l}
D^{\phi_{1}} U=\left(\mu_{B}+\alpha\right)(1-U-V)-\beta_{B} U Z, \\
D^{\phi_{2}} V=\beta_{B} U Z-\lambda_{B} V, \\
D^{\phi_{3}} Z=\beta_{T}(1-Z) V-\mu_{T} p Z .
\end{array}\right.
$$

Defined in the region $\Omega=\{(U, V, Z): 0 \leq U+V \leq 1,0 \leq Z \leq 1\}$, the sy $\mathrm{m}$ is cal $/$ commensurate if $\phi=\phi_{1}=\phi_{2}=\phi_{3}$; otherwise it is called incommensurate. The otic behavior of the system, when the total order of system is less than three is a it is connected to the fractal phase space in dynamics. Next variables of the babesiosis model living in $\Omega$ for all time $t \geq 0$. To establish our th result, we introduce the following lemma.

Lemma 1 (see [35]) Let the function $f \in C\left[{ }^{+}\right.$and its ractional derivative $D_{C}^{\varphi} f(t) \in$ $C\left[t_{0}, t_{1}\right]$ for $0 \leq \varphi<1$, and $t_{0}, t_{1} \in \mathbb{R}$; then or as

$$
f(t)=f\left(t_{0}\right)+\frac{1}{\Gamma(\varphi)} D_{C}^{\varphi} f(\tau)\left(t-t_{0}\right.
$$

for all $t \in\left(t_{0}, t_{1}\right]$, where $t_{0} \leq<<$.

Therefore, consider $\mathrm{g}$ the interval $\left[0, t_{1}\right]$ for any $t_{1}>0$, this theorem infers that the function $f:\left[0, t_{1}\right] \rightarrow \mathbb{K}$ no -increasing on $\left(0, t_{1}\right)$ if $D_{C}^{\varphi} f(t) \leq 0$ for all $t \in\left(0, t_{0}\right)$ and non-decreasing $\left.\quad t_{0}\right]$ if $D_{C}^{\varphi} f(t) \geq 0$ for all $t \in\left(0, t_{0}\right)$.

Preposicio. The region $\Omega=\{(U, V, Z): 0 \leq U+V \leq 1,0 \leq Z \leq 1\}$ is a positive invariant set for $s, \ldots, \ldots)$.

Pro By Theorem 3.1 and Remark 3.2 in [36], we obtain the global presence and rareness of the ucidations of (17).

We denote $\Omega_{+}=\{(U, V, Z): U \geq 0, V \geq 0, Z \geq 0\}$ if $(U(0), V(0), Z(0)) \in U$-axis = $\{(U, 0,0): U \geq 0\}$. Similarly, we can define $V$-axis and $Z$-axis. The vector field from (17) confined in $U$-axis assumes the form $F(U, V, Z)=\left(\left(\mu_{B}+\alpha\right)(1-U(t)), 0,0\right)$ by the Laplace transform properties (7), and we obtain the elucidation

$$
\begin{aligned}
& (U, V, Z) \\
& \quad=\left(t^{\varphi} E_{\varphi, \varphi+1}\left(-\left(\mu_{B}+\alpha\right) t^{\varphi}\right)\left(\mu_{B}+\alpha\right)+E_{\varphi, 1}\left(-\left(\mu_{B}+\alpha\right) t^{\varphi}\right) U(0), 0,0\right) \in U \text {-axis. }
\end{aligned}
$$

By the same argument, if $(U(0), V(0), Z(0)) \in V$-axis, we obtain

$$
(U, V, Z)=\left(0, E_{\varphi, 1}\left(-\lambda_{B} t^{\varphi}\right) V(0), 0\right) \in V \text {-axis, }
$$


and if $(U(0), V(0), Z(0)) \in Z$-axis, we have

$$
(U, V, Z)=\left(0,0, E_{\varphi, 1}\left(-\mu_{T} p t^{\varphi}\right) V(0)\right) \in V \text {-axis. }
$$

This proves that axes $U, V$, and $Z$ are solutions and positive invariant sets.

Now, we will prove that $\Omega_{+}$is a positive invariant set. By way of contradiction, suppose there exists a solution $(U, V, Z)$ such that $(U(0), V(0), Z(0)) \in \Omega_{+}$and the solution $(U, V, Z)$ to escape of $\Omega_{+}$. From the previous argument and by the unicity of solution $(U, V, Z)$ does not cross the axis. After the previous conclusion, there are three posc ilities.

(i) If the solution $(U, V, Z)$ escapes by the plane $U(t)=0$, then there existc $t_{0} \mathrm{~s}$ h that $U\left(t_{0}\right)=0, V\left(t_{0}\right)>0$, and $Z\left(t_{0}\right)>0$; and for all $t>t_{0}$ sufficiently near $t_{0}$, , have $U\left(t_{0}\right)<0$. Alternatively, $\left.D_{C}^{\varphi} U(t)\right|_{t=t_{0}}=\left(\mu_{B}+\alpha\right)\left(1-V\left(t_{0}\right)\right)>\left(\mu_{R} \quad\right)>0$. Fry $A$ Lemma 1, we obtain $U \geq U\left(t_{0}\right) \geq 0$ for all $t$ sufficiently near $t_{0}$, ana is not true.

(ii) If the solution $(U, V, Z)$ escapes by the plane $V(t)=0$, then $\mathrm{n}_{\mathrm{s}}$ ere exists $t_{0}$ such that $U\left(t_{0}\right)>0, V\left(t_{0}\right)=0$, and $Z\left(t_{0}\right)>0$; and for all $t>t_{0}$ suffli $u$, c $t_{0}$, we have $V\left(t_{0}\right)<0$. Alternatively, $\left.D_{C}^{\varphi} V(t)\right|_{t=t_{0}}=\beta_{B} U\left(t_{0}\right) Z(t>0$. Fro $\angle$ Cemma 1 , we obtain $V(t) \geq V\left(t_{0}\right) \geq 0$ for all $t$ sufficiently near $t_{0}$, and it is

(iii) If the solution $(U, V, Z)$ escapes by the plane $Z(t)=$, then there exists $t_{0}$ such that $U\left(t_{0}\right)>0, V\left(t_{0}\right)>0$, and $Z\left(t_{0}\right)=0$; and . IOr $>t_{0}$ sufficiently near $t_{0}$, we have $Z\left(t_{0}\right)<0$. On the other hand, $\left.D_{C}^{\varphi} Z(t)\right|_{t_{1}}=\beta_{T} /\left(t_{0}\right)>0$. From Lemma 1, we obtain $Z \geq Z\left(t_{0}\right) \geq 0$ for all $t$ sufficientiy near $t_{0}$, $\quad$ it is false.

Therefore, we obtain $U \geq 0, V \geq 0$, a $\quad \geq 0$ for all $t \geq 0$.

If $0 \leq U(0)+V(0) \leq 1$, from e first tr. equations of system (17), we get

$$
\begin{aligned}
D_{C}^{\varphi}(U(t)+V(t)) & =\left(\mu_{B}+\alpha\right)-\left(\mu_{B}+\alpha\right)(U(t)+V(t))-\lambda_{B} V(t) \\
& \left.=U_{B}+c\right)-\left(\mu_{B}+\alpha\right)(U(t)+V(t)) .
\end{aligned}
$$

Applying the Laplace trunsform in the previous inequality, we have

$$
\begin{gathered}
((L)+V(t))-\lambda^{\varphi-1}(U(0)+V(0)) \\
\leq \lambda^{1}\left(\mu_{B}+\alpha\right)-\left(\mu_{B}+\alpha\right) \mathcal{L}(U(t)+V(t)) .
\end{gathered}
$$

Til at can be written as

$$
\mathcal{L}(U(t)+V(t)) \leq\left(\mu_{B}+\alpha\right) \frac{\lambda^{\varphi-(1+\varphi)}}{\lambda^{\varphi}+\mu_{B}+\alpha}+\frac{\lambda^{\varphi-1}}{\lambda^{\varphi}+\mu_{B}+\alpha}(U(0)+V(0)) .
$$

From the Laplace transform properties (7) and (8), we infer that

$$
\begin{aligned}
(U(t)+V(t)) & \leq t^{\varphi} E_{\varphi, \varphi+1}\left(-\left(\mu_{B}+\alpha\right) t^{\varphi}\right)\left(\mu_{B}+\alpha\right)+E_{\varphi, 1}\left(-\left(\mu_{B}+\alpha\right) t^{\varphi}\right)(U(0)+V(0)) \\
& \leq t^{\varphi} E_{\varphi, \varphi+1}\left(-\left(\mu_{B}+\alpha\right) t^{\varphi}\right)\left(\mu_{B}+\alpha\right)+E_{\varphi, 1}\left(-\left(\mu_{B}+\alpha\right) t^{\varphi}\right)=1 .
\end{aligned}
$$

Therefore, we have that $0 \leq U(t)+V(t) \leq 1$. 
On the other hand, if $0 \leq Z(t) \leq 1$, from system (17) we obtain

$$
\begin{aligned}
D_{C}^{\varphi}(Z) & =\beta_{T}(1-Z) V-\mu_{T} p Z \\
& \leq\left(\beta_{T}+\mu_{T} p\right)(1-Z) .
\end{aligned}
$$

The proof of $0 \leq Z(t) \leq 1$ is similar to the previous case. Finally, we conclude that $\Omega$ is a positive invariant set.

\subsection{Existence and stability of equilibrium points}

There are two equilibrium points of system (17). Motivated by Aranda et al. [3] we will use the following threshold parameter. For more details on the threshold param ter, see $[37,38]$.

$$
R_{0}=\frac{\beta_{B} \beta_{T}}{\lambda_{B} \mu_{T} p}
$$

The value that $R_{0}$ yields can specify the situations in which, er $\quad ; z$ is likely. In the drug using context, $R_{0}$ tells us, on average, the total number o ople that each single drug user will initiate to drug use through the drug usin

\section{3 $\boldsymbol{R}_{\mathbf{0}}$ sensitivity analysis}

To examine the sensitivity of $R_{0}$ to each of facto

$$
\begin{aligned}
& \frac{\partial R_{0}}{\partial \beta_{B}}=\frac{\beta_{T}}{\lambda_{B} \mu_{T} p}>0, \\
& \frac{\partial R_{0}}{\partial \beta_{T}}=\frac{\beta_{B}}{\lambda_{B} \mu_{T} p}>0, \\
& \frac{\partial R_{0}}{\partial \lambda_{B}}=-\frac{\beta_{B} \beta_{T}}{\left(\lambda_{B}\right)^{2} \mu_{T}}<0, \\
& \frac{\partial R_{0}}{\partial \mu_{T}}=-\frac{1}{\lambda_{B}\left(l_{T} R_{T}\right.}<0, \\
& \partial P_{0}=\frac{\beta_{B} \beta_{T /}}{\partial{ }_{T}}<0 .
\end{aligned}
$$

T1 $R_{0}$ is increasing with $\beta_{B} \& \beta_{T}$ and is decreasing with $\lambda_{B}, \mu_{T} \& p$.

\subsection{Stability of DFE}

S. stem (17) has the DFE, i.e., $E_{0}=(1,0,0)$, for all the values of the factors in this system, whereas only if $R_{0}>1$, there is a (unique) EE point, i.e., $E_{1}=\left(U^{*}, V^{*}, Z^{*}\right)$, where

$$
\begin{aligned}
U^{*} & =\frac{\lambda_{B}\left\{\left(\mu_{B}+\alpha\right) \beta_{T}+p \mu_{T}\left(\mu_{B}+\alpha+\lambda_{B}\right)\right\}}{\beta_{T}\left\{\alpha\left(\beta_{B}+\lambda_{B}\right)+\mu_{B} \lambda_{B}+\beta_{B}\left(\mu_{B}+\lambda_{B}\right)\right\}}, \\
V^{*} & =\frac{\left(\mu_{B}+\alpha\right)\left(\beta_{B} \beta_{T}-\lambda_{B} \mu_{T} p\right)}{\beta_{T}\left\{\alpha\left(\beta_{B}+\lambda_{B}\right)+\mu_{B} \lambda_{B}+\beta_{B}\left(\mu_{B}+\lambda_{B}\right)\right\}}, \\
Z^{*} & =\frac{\left(\mu_{B}+\alpha\right)\left(\beta_{B} \beta_{T}-\lambda_{B} \mu_{T} p\right)}{\left(\mu_{B}+\alpha\right) \beta_{B} \beta_{T}+\left(\mu_{B}+\alpha+\lambda_{B}\right) \beta_{B} \mu_{T} p}
\end{aligned}
$$

in the interior of $\Omega$. 
The Jacobian matrix of system (17) is

$$
J=\left[\begin{array}{ccc}
-\left(\mu_{B}+\alpha\right)-\beta_{B} Z(t) & -\left(\mu_{B}+\alpha\right) & -\beta_{B} U(t) \\
\beta_{B} Z(t) & -\lambda_{B} & \beta_{B} U(t) \\
0 & \beta_{T}(1-Z(t)) & -\beta_{T} V(t)-\mu_{T} p
\end{array}\right]
$$

Now the Jacobian of system $(17)$ at the DFE $(1,0,0)$ is

$$
J\left(E_{0}\right)=\left[\begin{array}{ccc}
-\left(\mu_{B}+\alpha\right) & -\left(\mu_{B}+\alpha\right) & -\beta_{B} \\
0 & -\lambda_{B} & \beta_{B} \\
0 & \beta_{T} & -\mu_{T} p
\end{array}\right] .
$$

Consequently, the eigenvalues of $J\left(E_{0}\right)$ are

$$
\begin{aligned}
& \lambda_{1}=-\left(\mu_{B}+\alpha\right), \\
& \lambda_{2}=\frac{-\left(\lambda_{B}+\mu_{T} p\right)+\sqrt{\left(\lambda_{B}-\mu_{T} p\right)^{2}+4 \beta_{B} \beta_{T}}}{2}, \\
& \lambda_{3}=\frac{-\left(\lambda_{B}+\mu_{T} p\right)-\sqrt{\left(\lambda_{B}-\mu_{T} p\right)^{2}+4 \beta_{B} \beta_{T}}}{2} .
\end{aligned}
$$

It is easy to see that $\lambda_{2}$ and $\lambda_{3}$ are negative n $\mathrm{mb}$ If $R_{0}<1$, we observe

So,

$$
\begin{aligned}
\left(\lambda_{B}-\mu_{T} p\right)^{2}+4 \beta_{B} \beta_{T} & =\lambda_{B}^{2}+\mu_{T}^{2} p^{2}-2 \lambda_{B} \mu_{T T} \quad{ }_{4} \beta_{B} \beta_{T} \\
& <\lambda_{B}^{2}+\mu_{T}^{2} p^{2}-\lambda_{B} \mu_{\Gamma} p=\left(\lambda_{B}+\mu_{T} p\right)^{2} .
\end{aligned}
$$

$$
\lambda_{2}=\frac{-\left(\lambda_{B}+\mu_{T} p\right)}{\sqrt{\left(\lambda_{B}-\right.}-\underline{\left.\mu_{T} p\right)^{2}+4 \beta_{B} \beta_{T}}}
$$$$
<-\frac{-\left(\lambda_{B}+\lambda^{2} T /,\right.}{2} \frac{\sqrt{\left(\lambda_{B}+\mu_{T} p\right)^{2}}}{2}=0
$$

and

$$
\begin{aligned}
& -\frac{-\left(\lambda_{B}+\mu_{T} p\right)-\sqrt{\left(\lambda_{B}-\mu_{T} p\right)^{2}+4 \beta_{B} \beta_{T}}}{2} \\
& <\frac{-\left(\lambda_{B}+\mu_{T} p\right)-\sqrt{\left(\lambda_{B}+\mu_{T} p\right)^{2}}}{2}=-\left(\lambda_{B}+\mu_{T} p\right)<0 .
\end{aligned}
$$

Therefore $\lambda_{2}<0$ and $\lambda_{3}<0$; then we have that all the eigenvalues of the Jacobian matrix at $E_{0}$ are negative, i.e., $\left|\arg \left(\lambda_{i}\right)\right|=\pi, i=1,2,3$, and from Theorem 2, we have that the DFE point $E_{0}$ is locally asymptotically stable. Consequently, we have the following theorem.

Theorem 3 If $R_{0}<1$, then the disease-free point $E_{0}$ is locally asymptotically stable.

Now we will prove the global asymptotic stability of the DFE point.

Theorem 4 If $R_{0}<1$, then the disease-free point $E_{0}$ is worldwide asymptotically stable. 
Proof Suppose that $(U, V, Z)$ is the elucidation of system (17). Creating the variation of variables $M=1-U$, we obtain the new system

$$
\left\{\begin{array}{l}
D^{\phi_{1}} M=-\left(\mu_{B}+\alpha\right) M+\left(\mu_{B}+\alpha\right) V+\beta_{B} Z-\beta_{B} M Z \\
D^{\phi} V=\beta_{B}(1-M) Z-\lambda_{B} V \\
D^{\phi_{3}} Z=\beta_{T}(1-Z) V-\mu_{T} p Z .
\end{array}\right.
$$

It is easy to see that

$$
\begin{aligned}
& -\left(\mu_{B}+\alpha\right)(M-V)+\beta_{B}(1-M) Z \leq-\left(\mu_{B}+\alpha\right)(M-V)+\beta_{B} Z, \\
& \beta_{B}(1-M) Z-\lambda_{B} V \leq \beta_{B} Z-\lambda_{B} V \\
& \beta_{T}(1-Z) V-\mu_{T} p Z \leq \beta_{T} V-\mu_{T} p Z .
\end{aligned}
$$

From the above, it follows that the solutions $(M, V, Z)$ of syster (31) atisfy the differential inequality

$$
\left\{\begin{array}{l}
D^{\phi_{1}} M \leq-\left(\mu_{B}+\alpha\right)(M-V)+\beta_{B} Z \\
D^{\phi_{2}} V \leq \beta_{B} Z-\lambda_{B} V \\
D^{\phi_{3}} Z \leq \beta_{T} V-\mu_{T} P Z
\end{array}\right.
$$

Moreover, inspired by (33), let $(S, T, \quad)$ i the solution of the fractional linear system

$$
\left\{\begin{array}{l}
D^{\phi_{1}} S=-\left(\mu_{B}+\alpha\right)(S-\ulcorner)+, W \\
D^{\phi_{2}} T=\beta_{B} W-\lambda_{B} T \\
D^{\phi_{3}} W=\beta_{T} T-{ }_{-} W
\end{array}\right)
$$

with ICs $(S(0), T(P), y) \in \Omega$. The Jacobian of system (34) is

$$
J=\left[\begin{array}{ccc}
0 & -\left(\mu_{B}+\alpha\right) & -\beta_{B} \\
0 & -\lambda_{B} & \beta_{B} \\
0 & \beta_{T} & -\mu_{T} p
\end{array}\right] .
$$

To the Jacobian at the DFE is

$$
J\left(E_{0}\right)=\left[\begin{array}{ccc}
-\left(\mu_{B}+\alpha\right) & -\left(\mu_{B}+\alpha\right) & -\beta_{B} \\
0 & -\lambda_{B} & \beta_{B} \\
0 & \beta_{T} & -\mu_{T} p
\end{array}\right],
$$

and the eigenvalues of $J\left(E_{0}\right)$ are the same as derived above. Here we have proved that all the eigenvalues are negative. Thus $\left|\arg \left(\lambda_{i}\right)\right|=\pi, i=1,2,3$, and we can conclude that $\lim _{t \rightarrow \infty} S=0, \lim _{t \rightarrow \infty} T=0, \lim _{t \rightarrow \infty} W=0$. So, by Theorem 1 , we have $(M, V, Z) \leq \leq$ $(S, T, W)$. This implies that $\lim _{t \rightarrow \infty}(M, V, Z)=(0,0,0)$, and it follows that $(U, V, Z)$ converges to the DFE point $E_{0}=(1,0,0)$, when $R_{0}<1$. 


\subsection{Stability of EE}

Now we will show the local stability of the EE point $E_{1}$ with the help of some definitions $[39,40]$.

Definition 4 Let $Q$ be any matrix of real and complex numbers with order $n \times m$, let $q_{i_{1}, \ldots, j_{k}}$ be the minor of $A$ determined by the rows $\left(i_{1}, \ldots, i_{k}\right)$ and the columns $\left(j_{1}, \ldots, j_{k}\right)$, with $1 \leq i_{1}<i_{2}<\cdots<i_{k} \leq n$, and $1 \leq j_{1}<j_{2}<\cdots<j_{k} \leq m$. The $k$ th multiplicative compound matrix of $Q^{k}$ of $Q$ is the $\left(\frac{n}{k}\right) \times\left(\frac{n}{k}\right)$ matrix whose entries, written in a lexicographic order, ar $q_{i_{1}, \ldots, j_{k}}$. When $Q$ is an $n \times m$ matrix with columns $q_{1}, q_{2}, \ldots, q_{k}, Q^{k}$ is the exterior pro' uct $q_{1} \wedge q_{2} \wedge \cdots \wedge q_{k}$.

Definition 5 Let $Q=q_{i j}$ be an $n \times n$ matrix, its $k$ th additive compound ma. of $<$ of $Q$ is the $\left(\frac{n}{k}\right) \times\left(\frac{n}{k}\right)$ matrix given by $Q^{[k]}=\left|D(I+h Q)^{(k)}\right|=0$, where $D$ a differ - iation with respect to $h$. For any integers $i=1, \ldots,\left(\frac{n}{k}\right)$, let $(i)=\left(i_{1}, \ldots, i_{k}\right)$ be the $i$. ember in the lexicographic ordering of all $k$-tuples of integers such that $1 \leq i_{1}<\cdots<i_{k} \leq i_{n}$, then

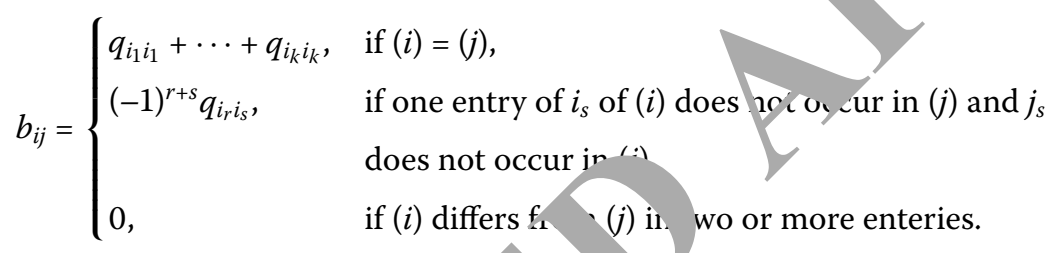

Remark 2 For $n=3$, the matrices $Q$ reas fllows:

$$
\begin{aligned}
& Q^{[1]}=Q, \quad Q^{[2]}=\left[\begin{array}{ccc}
q_{11}+q_{4} & q_{23} & -q_{13} \\
q_{32} & q_{22}+q_{33} & q_{12} \\
-q_{31} & q_{21} & q_{22}+q_{33}
\end{array}\right], \\
& Q^{[3]}=q_{11}+\quad+q_{33} .
\end{aligned}
$$

Lemm 2 t $t$ b a $a 3 \times 3$ real matrix. If $\operatorname{tr}(O)<0, \operatorname{det}(O)<0$, and $\operatorname{det}\left(O^{[2]}\right)<0$ are all negrtivt, en mo eigenvalues of $O$ have negative real parts.

Tht 5 If $R_{0}>1,\left(\mu_{B}+\alpha\right)>\beta_{T}$, and $\left(\mu_{B}+\alpha\right)>\beta_{B}$, then EE point $E_{1}$ is locally asymptoticaly stable.

Proof The Jacobian matrix of system (17) is given in (29).

From (29)

$$
\operatorname{tr}\left(J\left(E_{1}\right)\right)=-\left(\mu_{B}+\alpha\right)-\beta_{B} Z-\lambda_{B}-\beta_{T} V-\mu_{T} p<0 .
$$

To see that $\operatorname{det}\left(J\left(E_{1}\right)\right)<0$, we proceed as follows.

Since

$$
-\left(\mu_{B}+\alpha\right)=\frac{-\beta_{B} U Z}{(1-U-V)}, \quad \lambda_{B}=\frac{\beta_{B} U Z}{V}, \quad \mu_{T} p=\frac{\beta_{T}(1-Z) V}{Z},
$$


substituting (37) in (29), we have

$$
\operatorname{det}\left(J\left(E_{1}\right)\right)=\left|\begin{array}{ccc}
\frac{-\beta_{B} U Z}{(1-U-V)}-\beta_{B} Z & \frac{-\beta_{B} U Z}{(1-U-V)} & -\beta_{B} U \\
\beta_{B} Z & -\frac{\beta_{B} U Z}{V} & \beta_{B} U \\
0 & \beta_{T}(1-Z) & -\beta_{T} V-\frac{\beta_{T}(1-Z) V}{Z}
\end{array}\right| .
$$

On simplification

$$
\operatorname{det}\left(J\left(E_{1}\right)\right)=\left|\begin{array}{ccc}
\frac{\beta_{B} Z(1-V)}{(1-U-V)} & \frac{-\beta_{B} U Z}{(1-U-V)} & -\beta_{B} U \\
\beta_{B} Z & -\frac{\beta_{B} U Z}{V} & \beta_{B} U \\
0 & \beta_{T}(1-Z) & -\frac{\beta_{T} V}{Z}
\end{array}\right|
$$

We can easily see that $\operatorname{det}\left(J\left(E_{1}\right)\right)<0$, because all the parameters are $\mathrm{p} r$ ive cons its.

Now we will show that $\operatorname{det}\left(J^{[2]}\left(E_{1}\right)\right)<0$.

For this,

$$
\begin{aligned}
& \operatorname{det}\left(J^{[2]}\left(E_{1}\right)\right) \\
& =\left|\begin{array}{ccc}
-\left(\mu_{B}+\alpha\right)-\beta_{B} Z-\lambda_{B} & \beta_{B} U & \beta_{B} U \\
\beta_{T}-\beta_{T} Z & -\left(\mu_{B}+\alpha\right)-\beta \bar{z}-\beta_{T} V-\mu_{T} p & -\left(\mu_{B}+\alpha\right) \\
0 & \beta_{B}< & -\lambda_{B}-\beta_{T} V-\mu_{T} p
\end{array}\right|, \\
& \operatorname{det}\left(J^{[2]}\left(E_{1}\right)\right) \\
& \left.=-\left[\left(\left(\mu_{B}+\alpha\right)+\beta_{B} Z+\lambda_{B}\right)\left(\mu_{B}\right)+f_{B} Z+\beta_{T} V+\mu_{T} p\right)\left(\lambda_{B}+\beta_{T} V+\mu_{T} p\right)\right] \\
& +\beta_{T} \beta_{B} U(1-Z)\left[\beta_{L} Z++\beta_{T} V+\mu_{T} p\right]-\left(\left(\mu_{B}+\alpha\right)+\beta_{B} Z+\lambda_{B}\right)\left(\left(\mu_{B}+\alpha\right) \beta_{B} Z\right) \\
& \leq-\left[\left(\left(\mu_{B}+\alpha\right)+\beta_{B} Z+\lambda_{B}\right)\left(\left(\mu_{B}+\alpha\right)+\beta_{B} Z+\beta_{T} V+\mu_{T} p\right)\left(\lambda_{B}+\beta_{T} V+\mu_{T} p\right)\right] \\
& +\beta_{T} \beta_{B}\left[\beta_{B} Z+\beta_{\Gamma} V+\mu_{T} p\right]-\left(\left(\mu_{B}+\alpha\right)+\beta_{B} Z+\lambda_{B}\right)\left(\left(\mu_{B}+\alpha\right) \beta_{B} Z\right) \\
& =-\left(\lambda_{B}+\text { AT }_{T} p\right)\left[\left(\mu_{B}+\alpha+\beta_{B} Z+\lambda_{B}\right)\left(\mu_{B}+\alpha+\beta_{B} Z+\beta_{T} V+\mu_{T} p\right)-\beta_{T} \beta_{B}\right] \\
& -{ }_{B} Z\left[\left(\mu ;+\alpha+\lambda_{B}+\beta_{T} V\right)\left(\mu_{B}+\alpha\right)-\beta_{T} \beta_{B}\right] \text {. }
\end{aligned}
$$

lyzing $\mathrm{t}$ - terms of equality above, we have

$$
\begin{aligned}
& \left(\mu_{B}+\alpha+\beta_{B} Z+\lambda_{B}\right)\left(\mu_{B}+\alpha+\beta_{B} Z+\beta_{T} V+\mu_{T} p\right)>\beta_{T} \beta_{B} \\
& \left(\mu_{B}+\alpha+\lambda_{B}+\beta_{T} V\right)\left(\mu_{B}+\alpha\right)>\beta_{T} \beta_{B} .
\end{aligned}
$$

Then $\operatorname{det}\left(J^{[2]}\left(E_{1}\right)\right)<0$, and from Lemma 2 , the EE point $E_{1}$ is locally asymptotically stable. Hence the end of the proof of Theorem 5 .

\section{Numerical simulations}

In this section, we simulate different possible scenarios to check the effect that some values of fractional exponent $\phi$ have on the dynamics of bovine babesiosis disease and tick populations. For comparison purposes, we will use the same parameters as Aranda et al. [3]. 


\subsection{Adams-Bashforth-Moulton method}

For numerical elucidations of system (17), one can use the generalized Adams-BashforthMoulton method. To provide the estimated elucidation by means of this algorithm, consider the following nonlinear fractional differential equation [41]:

$$
\begin{aligned}
& D_{t}^{\alpha} y(t)=f(t, y(t)), \quad 0 \leq t \leq T, \\
& y^{(k)}(0)=y_{0}^{(k)}, \quad k=0,1,2, \ldots, m-1, \text { where } m=[\alpha] .
\end{aligned}
$$

The equation is equivalent to the Volterra integral equation

$$
y(0)=\sum_{k=0}^{m-1} y_{0}^{(k)} \frac{t^{k}}{k !}+\frac{1}{\Gamma(\alpha)} \int_{0}^{t}(t-s)^{\alpha-1} f(x, y(t)) d s .
$$

Diethelm et al. used the predictor-corrector scheme [42- 1, on the AdamsBashforth-Moulton algorithm to integrate (40). Also by settl $h=\frac{T}{N}, t_{n}=n h$, and $n=0,1,2, \ldots, N \in Z^{+}$, (17) can be discretized as follows [ $[+1$

$$
\begin{aligned}
& U^{n+1}=U(0)+\frac{h^{\phi_{1}}}{\Gamma\left(\phi_{1}+2\right)}\left(\left(\mu_{B}+\alpha\right)\left(1-{ }_{1}-,{ }_{1}\right)-\beta_{B} U_{n+1}^{p} Z_{n+1}^{p}\right) \\
& +\frac{h^{\phi_{1}}}{\Gamma\left(\phi_{1}+2\right)} \sum_{j=0}^{n} a_{j, n+1}\left(\mu_{\perp},\left(1-U_{j}-V_{j}\right)-\beta_{B} U_{j} Z_{j}\right), \\
& V^{n+1}=V(0)+\frac{h^{\phi_{2}}}{\Gamma\left(\zeta_{2}+2\right)}\left(\beta_{B} U_{n_{n}} \nexists_{n+1}^{p}-\lambda_{B} V_{n+1}^{p}\right) \\
& +\frac{h^{\varphi}}{\nabla\left(\phi_{2}+2\right)} \sum_{j=0}^{n} a_{j, n+1}\left(\beta_{B} U_{j} Z_{j}-\lambda_{B} V_{j}\right) \text {, } \\
& Z^{n+1}=(0)+\frac{h^{\phi_{3}}}{\Gamma\left(\phi_{3}+2\right)}\left(\beta_{T}\left(1-Z_{n+1}^{p}\right) V_{n+1}^{p}-\mu_{T} p Z_{n+1}^{p}\right) \\
& +\frac{h^{\phi_{3}}}{\Gamma\left(\phi_{3}+2\right)} \sum_{j=0}^{n} a_{j, n+1}\left(\beta_{T}\left(1-Z_{j}\right) V_{j}-\mu_{T} p Z_{j}\right)
\end{aligned}
$$

vere

$$
\begin{aligned}
& U_{n+1}^{p}=U(0)+\frac{1}{\Gamma\left(\phi_{1}\right)} \sum_{j=0}^{n} b_{j, n+1}\left(\left(\mu_{B}+\alpha\right)\left(1-U_{j}-V_{j}\right)-\beta_{B} U_{j} Z_{j}\right) \\
& V_{n+1}^{p}=V(0)+\frac{1}{\Gamma\left(\phi_{2}\right)} \sum_{j=0}^{n} b_{j, n+1}\left(\beta_{B} U_{j} Z_{j}-\lambda_{B} V_{j}\right) \\
& Z_{n+1}^{p}=Z(0)+\frac{1}{\Gamma\left(\phi_{3}\right)} \sum_{j=0}^{n} b_{j, n+1}\left(\beta_{T}\left(1-Z_{j}\right) V_{j}-\mu_{T} p Z_{j}\right)
\end{aligned}
$$


with

$$
a_{j, n+1}= \begin{cases}n^{\phi_{i}+1}-\left(n-\phi_{i}\right)(n+1)^{\phi_{i}}, & j=0, \\ (n-j+2)^{\phi_{i}+1}+(n-j)^{\phi_{i}+1}-2(n-j+1)^{\phi_{i}+1}, & 1 \leq j \leq n, \\ 1, & j=n+1,\end{cases}
$$

and

$$
b_{j, n+1}=\frac{h^{\phi_{i}}}{\phi_{i}}\left((n-j+1)^{\phi_{i}}-(n-j)^{\phi_{i}}\right), \quad 0 \leq j \leq n,
$$

with $i=1,2,3$.

\subsection{Disease-free equilibrium}

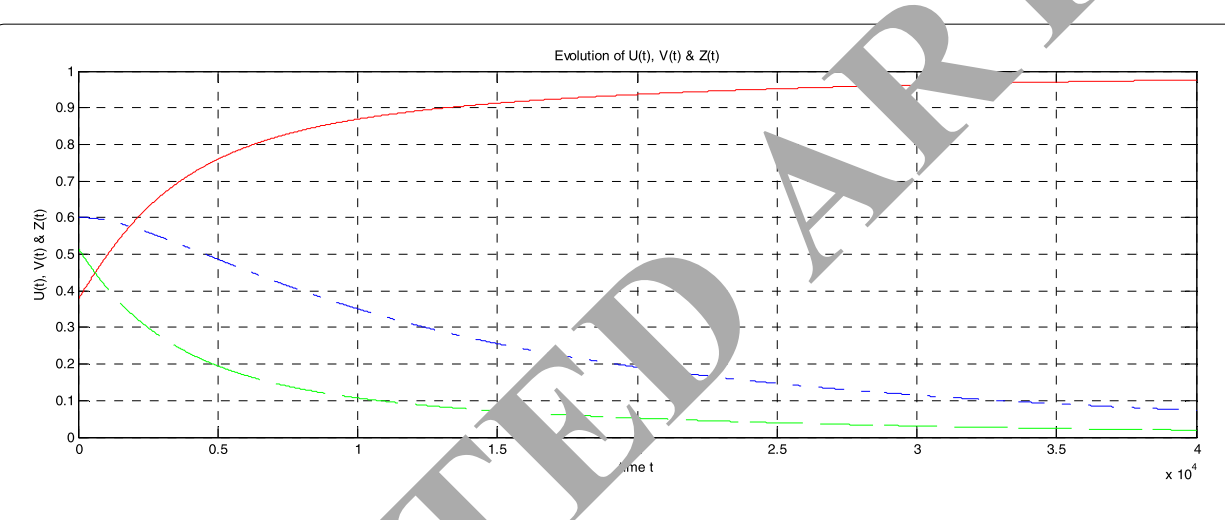

Figure 1 Dynamic of bovine batesios. "sease $U, V$, and $Z$ with $\phi_{1}=\phi_{2}=\phi_{3}=1$.

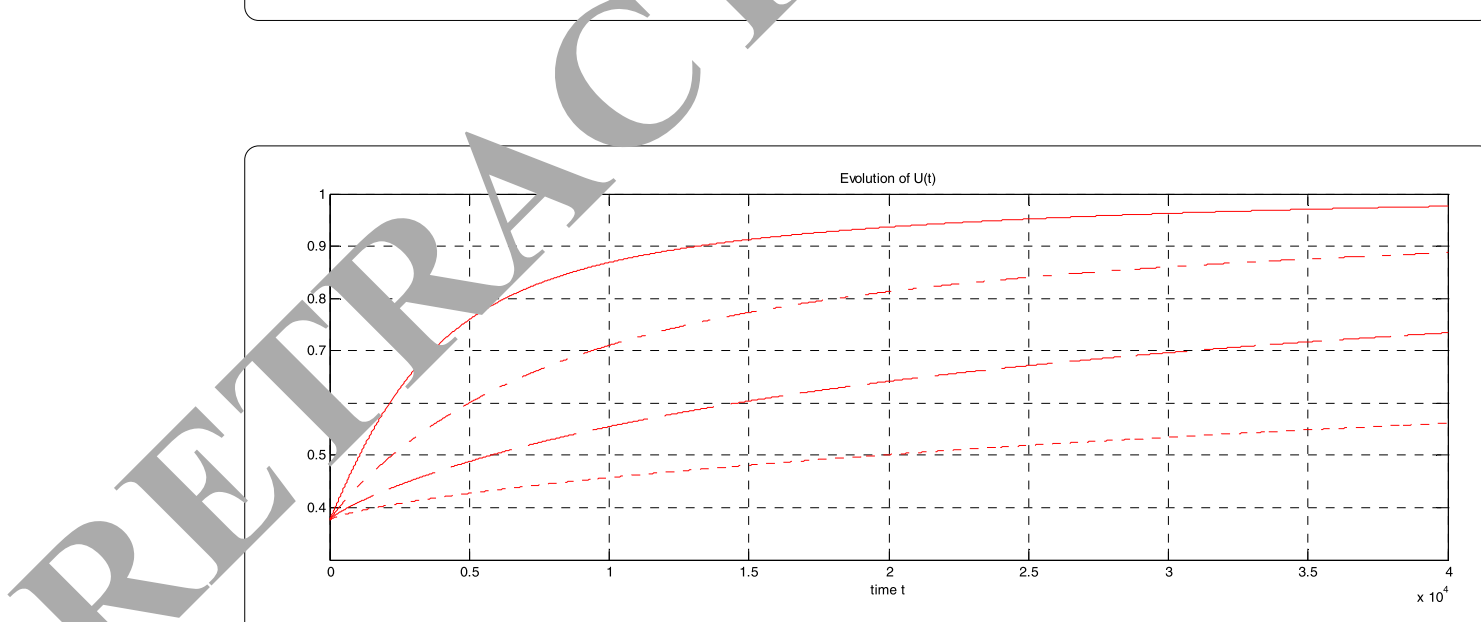

Figure 2 Dynamic of $U$ with $\phi_{1}=1$ (solid line), $\phi_{1}=0.9$ (dotted dashed line), $\phi_{1}=0.8$ (dashed line), and $\phi_{1}=0.7$ (dotted line). 


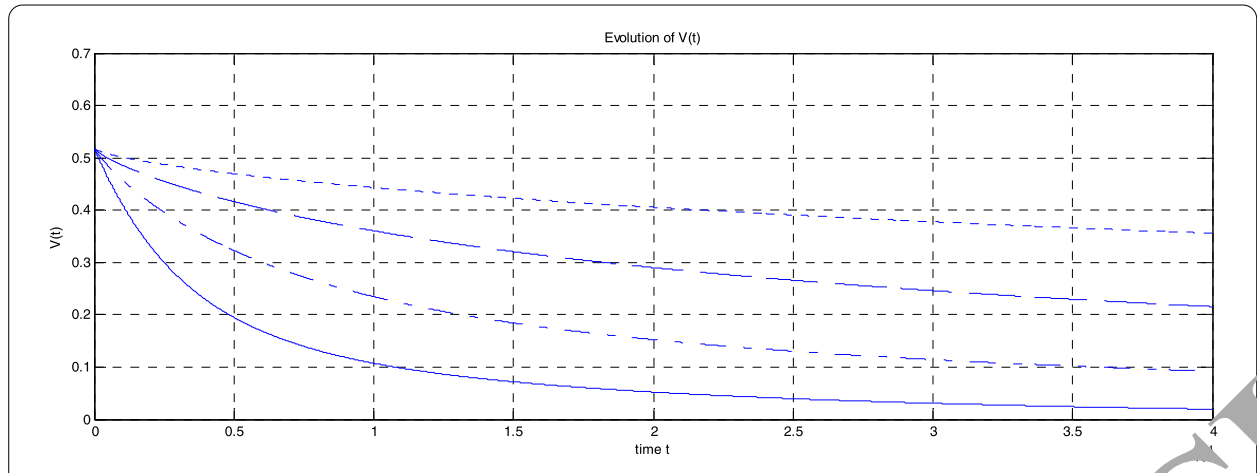

Figure 3 Dynamic of $V$ with $\phi_{2}=1$ (solid line), $\phi_{2}=0.9$ (dotted dashed line), $\phi_{2}=0.8$ (dashed ne), and $\phi_{2}=0.7$ (dotted line).

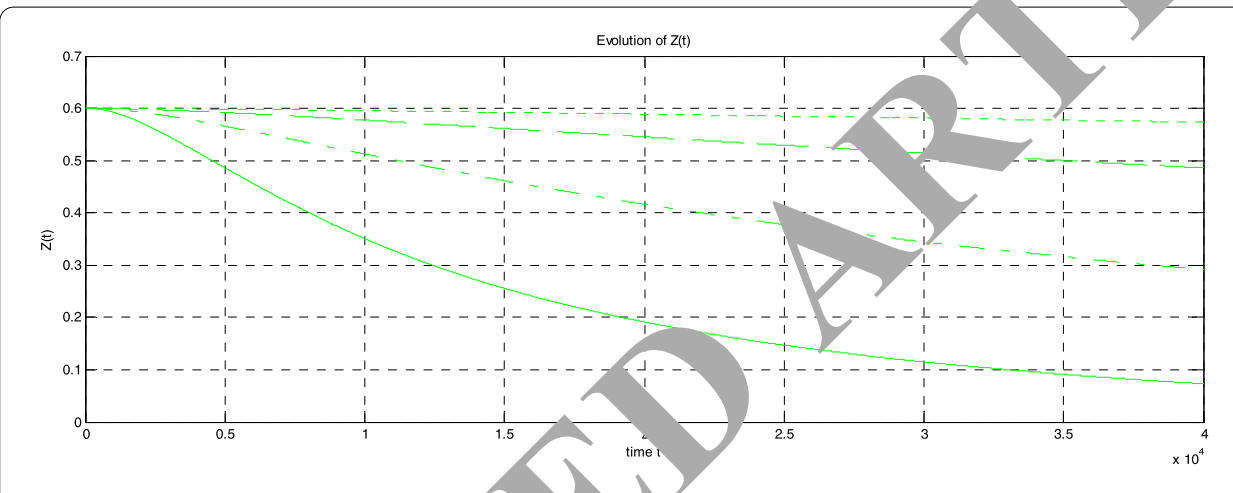

Figure 4 Dynamic of $Z$ with $\phi_{3}=1$ (s .... lin $\phi_{3}=c .9$ (dotted dashed line), $\phi_{3}=0.8$ (dashed line), and $\phi_{3}=0.7$ (dotted line).

\subsection{Endemic equilibi on}

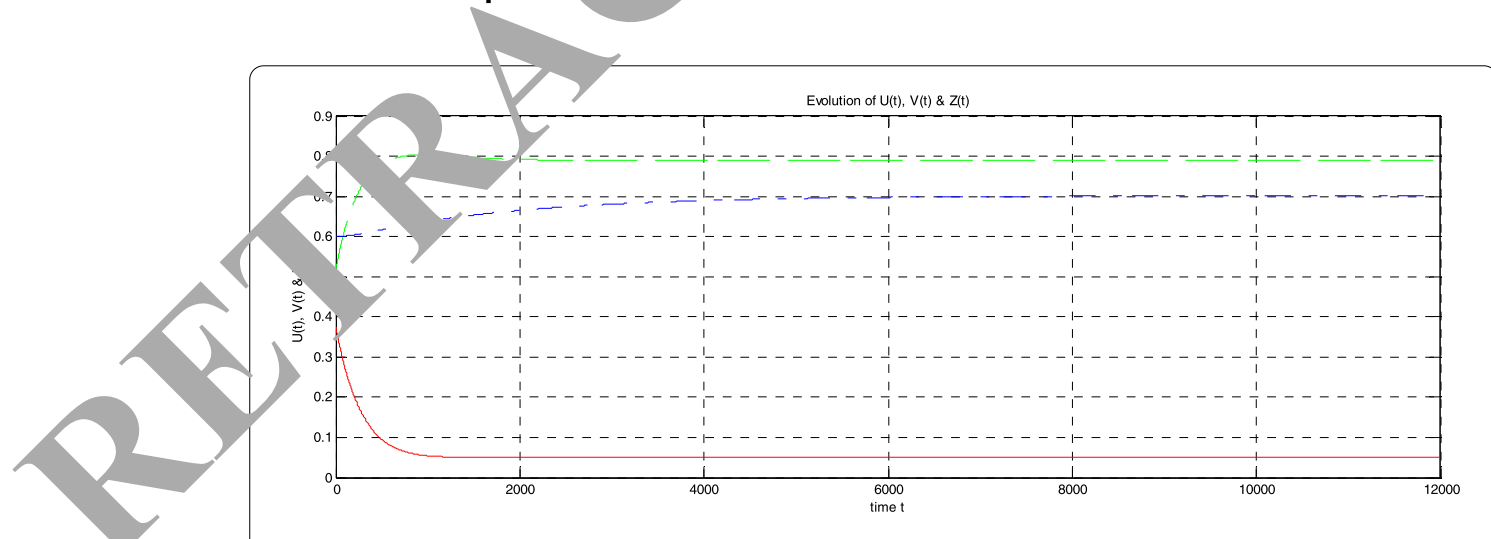

Figure 5 Dynamic of bovine babesiosis disease $U, V$, and $Z$ with $\phi_{1}=\phi_{2}=\phi_{3}=1$. 


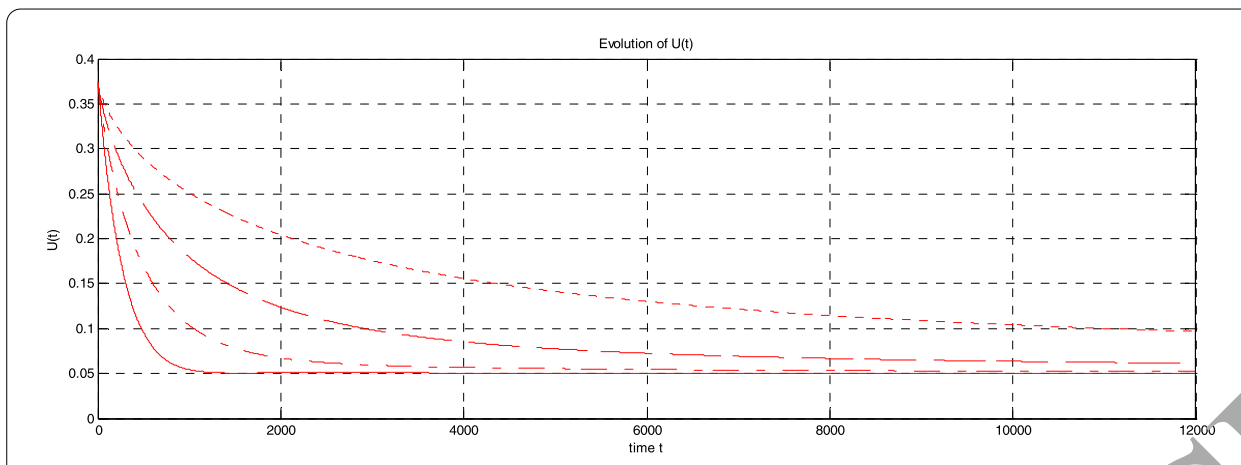

Figure 6 Dynamic of $U$ with $\phi_{1}=1$ (solid line), $\phi_{1}=0.9$ (dotted dashed line), $\phi_{1}=0.8$ (dashed ne) and $\phi_{1}=0.7$ (dotted line).

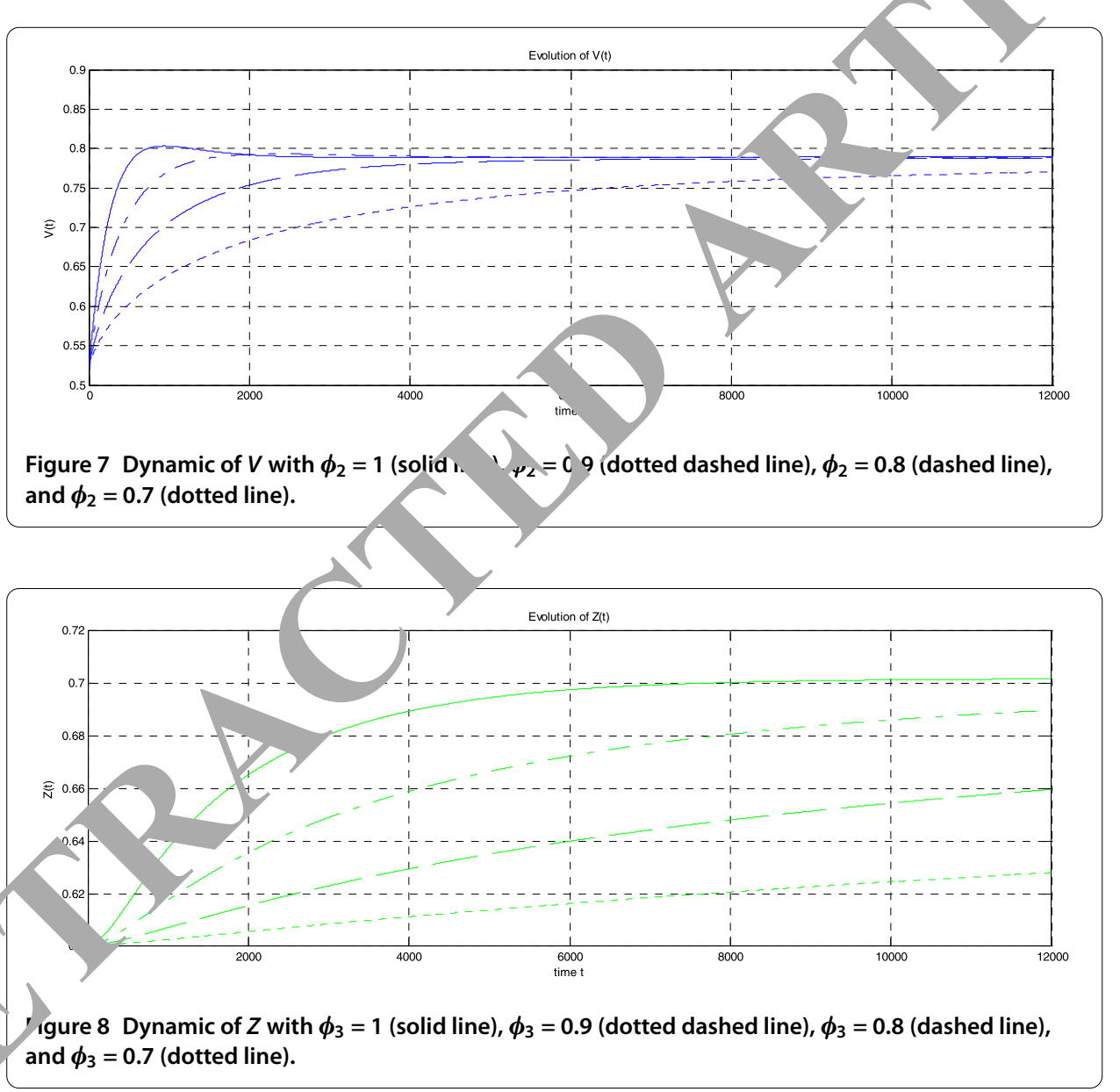

\section{Conclusions}

We have obtained the worldwide asymptotical stability of disease-free equilibrium using comparison theory of fractional differential equations since $R_{0}<1$. Therefore the proof that the endemic equilibrium point, when $R_{0}>1, \mu_{B}+\alpha>\beta_{B}$, and $\mu_{B}+\alpha>\beta_{T}$, is locally asymptotically stable was attained using the linearization theorem for fractional differential equations. Moreover, if $R_{0}<1$, then the system evolves to the endemic equilibrium 
point. To return to a disease-free status, the $R_{0}$ value should be greater than $1 . R_{0}<1$ is achieved when parameters $\beta_{B}$ and $\beta_{T}$ are very small or when parameters $\lambda_{B}, \mu_{T}$, and $p$ are very large. Therefore, a biological strategy to combat babesiosis disease would have to focus on one of these parameters. These results were confirmed by numerical simulations using the Adams-Bashforth-Moulton algorithm. Numerical simulations of an improved epidemic model with arbitrary order have shown that fractional order is related to relaxation time, in other words, the time taken to reach equilibrium. The chaotic behavior of the system when the total order of system is less than three is sketched. A compariso between four different values of the fractional order is shown in Figures $1,2,3$, and 4 , vitı. the same control parameter as $\mu_{B}=0.0002999, \alpha=0.001, \beta_{B}=0.006, \lambda_{B}=0.500265$, $\beta_{T}=0.00048, \mu_{T}=0.001609, p=0.1$. Figures $1,2,3$, and 4 show different beha ors for $\phi=0.7, \phi=0.8, \phi=0.9$, and $\phi=1$. For all four cases, the disease evolves $\pi$ free equilibrium point and endemic equilibrium point; however, it is sle ver whe. $y=0.9$, when $\phi=0.8$, it is slower than $\phi=0.9$. And it is much slower whe $\phi \quad 7$. Numerical simulations with different order show that the system decays to f librium Jndition like power law $t^{-\phi}$, as previously established in [45]. This result p. ide m important insight about the use of fractional order to model the dynamics of ba iosis disease and tick population. The proof shown here should be used as a in the study of equilibrium conditions in similar problems, such as tuberculosis [46], nalaria [47], or toxoplasmosis disease [48].

Competing interests

The authors declare that they have no competin

Authors' contributions

The authors have achieved equal contrisun All authos read and approved the final version of the manuscript.

Author details

'Department of Mathematics,' niversity of Engireering and Technology, Lahore, Pakistan. ${ }^{2}$ Faculty of Information Technology, University of Cent Punjab, La hore, Pakistan. ${ }^{3}$ Department of Mathematics, University of Engineering and Technology, KSK Campus, Laho akistan

\section{Acknowledgements}

We would like to thank the ete., es for their valuable comments.

Publís,

Sp nger Natu. emains neutral with regard to jurisdictional claims in published maps and institutional affiliations.

Rece $\quad$ : 3 November 2016 Accepted: 6 March 2017 Published online: 22 March 2017

References

- Benavides, E: Considerations with respect to the epizootilogia of an aplasmosis and babesiosis in the bovines ACOVEZ 31, 4-11 (1985)

. Kermack, WO, McKendrick, AG: A contribution to the mathematical theory of epidemics. Proc. R. Soc. A, Math. Phys. Eng. Sci. 115, 700-721 (1927)

3. Aranda, DF, Trejos, DY, Valverde, JC, Villanueva, RJ: A mathematical model for babesiosis disease in bovine and tick populations. Math. Methods Appl. Sci. 35(3), 249-256 (2012)

4. Keeling, MJ, Eames, KTD: Networks and epidemic models. J. R. Soc. Interface 2(4), 295-307 (2005)

5. Wang, Y, Jin, Z, Yang, Z, Zhang, ZK, Zhou, T, Sun, GQ: Global analysis of an SIS model with an infective vector on complex networks. Nonlinear Anal., Real World Appl. 13(2), 543-557 (2012)

6. Wang, Y, Cao, J: Global dynamics of a network epidemic model for waterborne diseases spread. Appl. Math. Comput. 237, 474-488 (2014)

7. Caputo, M: Lectures on seismology and rheological tectonics. Lecture Notes, Dipartimento di Fisica, Università La Sapienza, Roma, Italy (1992)

8. Ciesielski, M, Leszczynski, J: Numerical simulations of anomalous diffusion. In: CMM (2003). http://arxiv.org/abs/math-ph/0309007

9. Demirci, E, Ozalp, N: A method for solving differential equations of fractional order. J. Comput. Appl. Math. 236(11), 2754-2762 (2012) 
10. Diethelm, K: The Analysis of Fractional Differential Equations: An Application-Oriented Exposition Using Operators of Caputo Type. Springer, Berlin (2004)

11. Du, M, Wang, Z, Hu, H: Measuring memory with the order of fractional derivative. Sci. Rep. 3, 3431 (2013)

12. Li, C, Ma, Y: Fractional dynamical system and its linearization theorem. Nonlinear Dyn. 71(4), 621-633 (2013)

13. Lorenzo, CF, Hartley, TT: Initialization, conceptualization, and application in the generalized (fractional) calculus. Crit. Rev. Biomed. Eng. 35(6), 447-553 (2007)

14. McCall, PJ, Kelly, DW: Learning and memory in disease vectors. Trends Parasitol. 18(10), 429-433 (2002)

15. Wang, Z, Yang, D, Ma, T, Sun, N: Stability analysis for nonlinear fractional-order systems based on comparison principle. Nonlinear Dyn. 75(1-2), 387-402 (2014)

16. González-Parra, G, Arenas, AJ, Chen-Charpentier, BM: A fractional order epidemic model for the simulation of outbreaks of influenza A(H1N1). Math. Methods Appl. Sci. 37(15), 2218-2226 (2014)

17. Pooseh, S, Rodrigues, H, Torres, D: Fractional derivatives in dengue epidemics. AIP Conf. Proc. 1389, 739-742 (2011)

18. Diethelm, K: A fractional calculus based model for the simulation of an outbreak of dengue fever. Nonlinear Dyn 71(4), 613-619 (2013)

19. Sardar, T, Rana, S, Chattopadhyay, J: A mathematical model of dengue transmission with memory. Commu Nonlinear Sci. Numer. Simul. 22(1-3), 511-525 (2015)

20. Baleanu, D, Golmankhaneh, AK, Golmankhaneh, AK, Nigmatullin, RR: Newtonian law with memory. Nonlir Dyn. 60, 81-86 (2010)

21. Baleanu, D, Mustafa, OG: On the global existence of solutions to a class of fractional differential quat, Comput. Math. Appl. 59, 1835-1841 (2010)

22. Agila, A, Baleanu, D, Eid, R, Irfanoglu, B: Applications of the extended fractional Euler-Lagrang ruations m, odel to freely oscillating dynamical systems. Rom. J. Phys. 61(3-4), 350-359 (2016)

23. West, BJ, Grigolini, P, Metzler, R, Nonnenmacher, TF: Fractional diffusion and Le'vy st+ processes. ys. Rev. E 55(1), 99-106 (1997)

24. Metzler, R, Klafter, J: The random walk's guide to anomalous diffusion: a fractior 1-77 (2000)

25. Golmankhaneh, AK, Arefi, R, Baleanu, D: Synchronization in a nonidenticl fractional o of a proposed modified system. J. Vib. Control 21(6), 1154-1161 (2015)

26. Purohit, SD, Kalla, SL: On fractional partial differential equations related to qurntu. mechanics. J. Phys. A, Math. Theor. 44(4), 045202 (2011)

27. Purohit, SD: Solutions of fractional partial differential equati f quantum nechanics. Adv. Appl. Math. Mech. 5(5), 639-651 (2013)

28. Chouhan, A, Purohit, SD, Saraswat, S: An alternate metr. for solv generalized differential equations of fractional order. Kragujev. J. Math. 37(2), 299-306 (2013)

29. Nisar, KS, Purohit, SD, Mondal, SR: Generalized actional kine, quations involving generalized Struv function of the first kind J. King Saud Univ Sci. 28(2) 167-1, 01 ,

30. Baleanu, D, Diethelm, K, Scalas, E, Trujillo,....ract. I Calc alus: Models and Numerical Methods, vol. 3. World Scientific, Singapore (2012)

31. Zafar, Z, Ahmad, MO, Pervaiz, A, R- q, M urth order compact method for one dimensional inhomogeneous telegraph equation with $O\left(h^{4}, k^{2}\right.$. Pak. J. E. $\quad$ pol. Sci. 14, 96-101 (2014)

32. Zafar, Z, Rehan, K, Mushtaq M, Rafiq, M: Num, ical modeling for nonlinear biochemical reaction networks. IJMC (Accepted manuscript)

33. Zafar, Z, Rehan, K, Mushta 1, Rafiq, M Numerical treatment for nonlinear Brusselator chemical model. J. Differ. Equ. Appl. (2016). doi:10.1080/1 198 2 16.1257005 (online on 21st Nov, 2016)

34. Podlubny, I: Fracti ol derivatives. history, theory, application. Utah State University, Logan, Utah, USA (2005)

35. Odibat, ZM, Shawa gio Coneralized Taylor's formula. Appl. Math. Comput. 186(1), 286-293 (2007)

36. Lin, W: Global existe ic the, ry and chaos control of fractional differential equations. J. Math. Anal. Appl. 332(1), 709-7201

37. Cin+ -Aria A Casti,o-Chávez, C, Bettencourt, LM, Lloyd, AL, Banks, HT: The estimation of the effective reproductive numb ontur se outbreak data. Math. Biosci. Eng. 6(2), 261-282 (2009)

38 Dietz, K: sstimation of the basic reproduction number for infectious diseases. Stat Methods Med. Res. 2(1), 23-41 1993)

39. h, K, Otoo, L, Hayes, RJ, Carson, DC, Greenwood, BM: Antibodies to blood stage antigens of Plasmodium far $u m$ in rural Gambians and their relation to protection against infection. Trans. R. Soc. Trop. Med. Hyg. 83(3), 293) $303(1989)$

40. Tumwiine, J, Mugisha, JYT, Luboobi, LS: A mathematical model for the dynamics of malaria in a human host and mosquito vector with temporary immunity. Appl. Math. Comput. 189(2), 1953-1965 (2007)

1. Li, C, Tao, C: On the fractional Adams method. Comput. Math. Appl. 58(8), 1573-1588 (2009)

42. Diethelm, K: An algorithm for the numerical solution of differential equations of fractional order. Electron. Trans. Numer. Anal. 5, 1-6 (1997)

43. Diethelm, K, Ford, NJ: Analysis of fractional differential equations. J. Math. Anal. Appl. 265(2), 229-248 (2002)

44. Diethelm, K, Ford, NJ, Freed, AD: A predictor-corrector approach for the numerical solution of fractional differential equations. Nonlinear Dyn. 29(1-4), 3-22 (2002)

45. Matignon, D: Stability results for fractional differential equations with applications to control processing. Comput. Eng. Syst. Appl. 2, 963-968 (1996)

46. McCluskey, C, Van den Driessche, P: Global analysis of tuberculosis models. J. Differ. Equ. 16, 139-166 (2004)

47. Ngwa, GA, Shu, WS: A mathematical model for endemic malaria with variable human and mosquito populations. Math. Comput. Model. 32(7-8), 747-763 (2000)

48. González-Parra, GC, Arenas, AJ, Aranda, DF, Villanueva, RJ, Jódar, L: Dynamics of a model of toxoplasmosis disease in human and cat populations. Comput. Math. Appl. 57(10), 1692-1700 (2009) 